\title{
Nonverbal Behavior in the Digital Age: Meanings, Models, and Methods
}

\author{
Joann M. Montepare
}

Published online: 18 May 2014

(C) Springer Science+Business Media New York 2014

\begin{abstract}
This Special Issue of the Journal of Nonverbal Behavior explores nonverbal issues in the digital age, with an emphasis how digital technologies provide researchers with new tools for exploring nonverbal components of interpersonal interactions in digital environments. In addition to showing a range of exciting applications, the featured articles also touch upon the challenges of new digital strategies.
\end{abstract}

Keywords Nonverbal behavior · Digital environment $\cdot$ New technologies

Predictions about the coming of the digital age and assumptions about how technology will transform our lives have served as imaginative content in science fiction for decades. Today we see such speculations rapidly coming to fruition in diverse contexts. This Special Issue of the Journal of Nonverbal Behavior offers a glimpse of ways in which contemporary digital developments are now part of nonverbal scientists' toolkit for examining the components of nonverbal behavior in greater depth.

The haunting voice of Hal in 2001: A Space Odyssey (1968) uttering "I'm sorry, Dave. I'm afraid I can't do that" left movie viewers wondering if computers would one day be able to assume human emotions. While it remains to be seen if computers can indeed assume such qualities, research by Balzarotti et al. (2014) reported here suggests that individuals may nevertheless believe that they do, and respond in predictable ways to affective human-computer interactions. When playing a game with a computer that simulated understanding of their emotional reactions while guiding them through game activities, participants reported a sense of awareness of interacting with an agent able to recognize their emotions and "understand" them. Moreover, participants responded nonverbally to the affective human-computer interactions. It is not hard to conceive of avenues for future research in this realm. Certainly knowing more about the nature of digital

J. M. Montepare (ه)

RoseMary B. Fuss Center for Research on Aging and Intergenerational Studies, Lasell College, 1844

Commonwealth Avenue, Newton, MA 02466, USA

e-mail: jmontepare@lasell.edu 
(non)verbal information sufficient to elicit emotional attunements is of interest, as is comparing the nature of emotional responses in mediated and non-mediated contexts. Of particular interest will be research examining the translation of knowledge gained from the empirical study of nonverbal human-computer interfaces to applications in educational, medical, entertainment, and other digital contexts.

Haans et al. (2014) provide new information and raise further questions about applications in technological developments in their study which moves beyond the more typical digital focus on auditory and visual information to interpersonal physical contact-touch. In a virtual demonstration of the "Midas Touch" phenomenon, these researchers show that the touch effect is also operative in mediated interactions where the touch act is replaced by electromechanical stimulation through a tactile display. In addition to having implications for the design of communication media, which has not yet integrated physical contact, their review of previous nonverbal research on touch also suggests the promising use of tactile displays in future work to avoid the confederate bias inherent in studies in naturalistic settings.

The implications of digital technology in human interaction are examined further in Patterson et al. (2014) timely exploration of the effects of mobile device use on pedestrian encounters. Complementing recent survey reports of an increase in the number of physical accidents associated with cellphone-using adults who report bumping into another person or an object while talking or texting on their phone (Pew 2014), these researchers demonstrate the social implications of cell-use in an experimental field setting. Specifically, when a confederate passerby displayed a greeting toward a person using versus not using a cell phone, mobile-device users showed a significant reduction in responsiveness. Gender differences were also observed, with significantly fewer female users offering greetings than males, raising questions about the underlying mechanisms which drive social outcomes associated with the use of mobile digital technologies.

The digital age also has ushered in powerful computer-based methods for analyzing highly elusive patterns of nonverbal behavior, as demonstrated by several papers in this Special Issue. In their study of patterns of nonverbal behavior associated with truth and deception, Burgoon et al. (2014) show us how the software program THEME has the potential to discover patterns among discrete events in time-ordered data that characterize deceptive individuals in social interactions. In their study of patterns of nonverbal behavior associated with creativity in collaborating dyads, Won et al. (2014) track the nonverbal behavior of dyad members engaging in a creativity task using the Microsoft Kinect computer vision algorithm and reveal differences in nonverbal synchrony displayed by dyads whose work was evaluated as highly creative. In addition to showing the powerful utility of computer systems for capturing and evaluating complex nonverbal activity, this research paves the way not only for the study of other elusive behaviors, but also for the discovery of new nonverbal actions and patterns.

The final papers featured in this Special Issue remind us of the limitations and challenges of working in a digital environment. Dunbar et al. (2014) found that video-conferencing hampered the interactional synchrony of members of an interacting dyad, especially when one of the participants was accused of cheating. In addition to providing insights about detecting mediated and non-mediated deception, this work raises serious questions about the extent to which nonverbal dynamics may be disrupted and jeopardized in an environment which is fast becoming more common for professional, educational, medical, social, and other interactions. In their study of the utility of using video images and speech-signal analysis in dyadic conversation to analyze hand gestures, adaptors, and utterances, Fujiwara and Daibo (2014) found promising outcomes, although differences 
were observed depending on nonverbal activity. Thus, the advantage of computer-based methods over traditional manual methods requires more scrutiny. Researchers point out that present systems face a number of challenges, including the fact that they are restricted primarily to settings where recordings can be easily set up and to interactions which are fairly structured and relatively simple. As well, many systems are not yet openly available to the nonverbal research community and require special training for use and analysis. However, we hope that the work featured in this Special Issue convinces readers of the great strides being made, and that the return on digital investments has great capacity to be significant-as researchers explore new frontiers and boldly go where no nonverbal researcher has gone before!

\section{References}

Balzarotti, S., Piccini, L., Andreoni, G., \& Ciceri, R. (2014). "I know that you know how I feel": Behavioral and physiological signals demonstrate emotional attunement interacting with a computer simulating emotional intelligence. Journal of Nonverbal Behavior. doi:10.1007/s10919-014-0180-6.

Burgoon, J. K., Proudfoot, J. G., Schuetzler, R., \& Wilson, D. (2014). Patterns of nonverbal behavior associated with truth and deception: Illustrations from three experiments. Journal of Nonverbal Behavior. doi:10.1007/s10919-014-0181-5.

Dunbar, N. E., Jensen, M. L., Tower, D. C., \& Burgoon, J. K. (2014). Synchronization of nonverbal behaviors in detecting mediated and non-mediated deception. Journal of Nonverbal Behavior. doi:10. 1007/s10919-014-0179-z.

Fujiwara, K. \& Daibo, I. (2014). The extraction of nonverbal behaviors: Using video images and speech-signal analysis in dyadic conversation. Journal of Nonverbal Behavior. doi:10.1007/s10919-014-0183-3.

Haans, A., de Bruijn, R., \& IJsselsteijn, W. A. (2014). A virtual Midas touch? Touch, compliance, and confederate bias in mediated communication. Journal of Nonverbal Behavior. doi:10.1007/s10919014-0184-2.

Patterson, M. L., Lammers, V. M., \& Tubbs, M. E. (2014). Busy signal: Effects of mobile device usage on pedestrian encounters. Journal of Nonverbal Behavior. doi:10.1007/s10919-014-0182-4.

Pew Research Center. (2014, January 2). More than half of cell owners affected by "distracted walking". Retrieved from http://www.pewtrusts.org/our_work_report_detail_full.aspx?id=85899531452.

Won, A. S., Dai, W. \& Bailenson, J. N. (2014). Automatically detected nonverbal behavior predicts creativity in collaborating dyads. Journal of Nonverbal Behavior. doi:10.1007/s10919-014-0186-0. 